\title{
Effects of bone marrow-derived mesenchymal stem cells transfected with survivin on pulmonary fibrosis in mice
}

\author{
MI ZHOU*, DONG-LING CHEN*, TAO JIANG, YAN-MEI FENG and XIAO-LI HAN \\ Department of Respiratory Medicine, The First Affiliated Hospital of Chongqing Medical University, \\ Yuzhong, Chongqing 400042, P.R. China
}

Received August 26, 2014; Accepted July 17, 2015

DOI: $10.3892 /$ etm.2015.2715

\begin{abstract}
The aim of the present study was to investigate the effects of bone marrow-derived mesenchymal stem cells (BMSCs) transfected with survivin on lung fibrosis in mice. Mice with bleomycin-induced pulmonary fibrosis were allocated at random to group A, B or C, and injected with $1 \times 10^{6}$ survivin gene-expressing BMSCs, $1 \times 10^{6}$ BMSCs or normal saline, respectively. A total of 6 mice were sacrificed from each group on days 7, 14 and 28 after treatment. The extent of alveolitis and pulmonary fibrosis was assessed and the apoptotic rates of the BMSCs and survivin-expressing BMSCs were detected. The content of surfactant protein A (SP-A) in the lung and hydroxyproline (Hyp) in the serum was measured. The mRNA expression levels of transforming growth factor (TGF)- $\beta 1$ and matrix metalloproteinase (MMP)-9 in the lung tissue of the mice was detected. Furthermore, the protein expression levels of caspase- 3 and -9 were detected. The apoptotic rates of the BMSCs (group B) and survivin-expressing BMSCs (group A) were $14.466 \pm 1.953$ and $7.718 \pm 0.493 \%$, respectively. The degree of lung fibrosis in groups A and B was reduced compared with that in group $\mathrm{C}$. The hydroxyproline content in groups A and $\mathrm{B}$ was reduced compared with that in group $\mathrm{C}$, and the SP-A content in groups A and B was increased compared with that in group C. The mRNA expression levels of TGF- $\beta 1$ in group A were reduced compared with those in group B, and the levels in group B were reduced compared with those in group C. By contrast, the mRNA expression levels of MMP-9 in group A were increased compared with those in groups B and $\mathrm{C}$, and the levels in group B were increased compared with those in group A. The expression levels of caspase-3 and -9
\end{abstract}

Correspondence to: Professor Tao Jiang, Department of Respiratory Medicine, The First Affiliated Hospital of Chongqing Medical University, 1 Youyi road, Yuzhong, Chongqing 400042, P.R. China

E-mail: jiangtao1341@yeah.net

${ }^{*}$ Contributed equally

Key words: mesenchymal stem cells, survivin, pulmonary fibrosis, mice in group A were elevated compared with those in groups B and $\mathrm{C}$. In conclusion, BMSCs are effective in preventing bleomycin-induced lung fibrosis, and survivin may enhance the protective effects of BMSCs.

\section{Introduction}

Pulmonary fibrosis is a progressive and fatal disease characterized by pulmonary interstitial fibrosis, which may result in diffuse alveolar inflammation and structural disorder with decreasing lung volumes and hypoxemic respiratory failure $(1,2)$. Despite intense research efforts, there are currently no effective therapies to reduce the high fatality rate of pulmonary fibrosis (3).

Bone marrow-derived mesenchymal stem cells (BMSCs) are easy to separate, purify and augment in vitro, and are targeted to and differentiate in damaged tissue. Furthermore, BMSCs are able to regulate immune function and promote the expression of various growth factors locally in the damaged tissue, thus reducing inflammation and collagen deposition and potentially exerting a therapeutic effect against lung fibrosis $(4,5)$.

Survivin is a member of the apoptosis inhibitory protein family, and exerts an antiapoptotic effect primarily by inhibiting the activity of cysteine-aspartic acid protease-3 (caspase-3) (6). Lentiviruses are among the most commonly used vectors of gene therapy, possessing high transfection efficiency and a high level of exogenous gene expression. Green fluorescent protein (GFP) is characterized by stability, high efficiency and non-toxicity and is easy to detect. GFP can used to observe living cells directly and to trace stem cells in vivo. In the present study, BMSCs containing GFP and the survivin gene were introduced into a bleomycin-induced mouse model of pulmonary fibrosis. The effect of BMSCs and survivin on the pulmonary fibrosis was then assessed.

\section{Materials and methods}

Reagents and kits. BMSCs were obtained from Cyagen Biosciences, Inc. (Santa Clara, CA, USA). Cell culture medium and fetal bovine serum (FBS) were purchased from Gibco Life Technologies (Carlsbad, CA, USA). Bleomycin hydrochloride for injection was purchased from Nippon Kayaku Co., Ltd. (Tokyo, Japan). An hydroxyproline ELISA detection kit was 
purchased from Wuhan ColorfulGene Biological Technology Co., Ltd. (Wuhan, China). Quantitative polymerase chain reaction ( $\mathrm{qPCR}$ ) reagents were obtained from Takara Biotechnology Co., Ltd. (Dalian, China), rabbit anti-mouse surfactant protein A (SP-A) immunohistochemistry kits from Wuhan Boster Biological Technology, Ltd. (Wuhan, China) and Beyotime Institute of Biological Technology (Haimen, China). Secondary antibodies for western blot analysis were purchased from Santa Cruz Biotechnology, Inc. (Dallas, TX, USA).

Animals. All procedures in this study were approved by the Animal Studies Committee of Chongqing Medical University (Chongqing, China) and were conducted in accordance with the Guide for the Care and Use of Laboratory Animals published by the National Institutes of Health. Male C57BL/6 mice, 6-8 weeks old and weighing $22 \mathrm{~g}$, were supplied by the animal center of Chongqing Medical University and were housed in a specific pathogen-free facility.

BMSC culture. This study was conducted in the Respiratory Department of the First Affiliated Hospital of Chongqing Medical University. BMSCs derived from C57BL/6 mice were cultured in F12-Dulbecco's modified Eagle's medium (DMEM) medium containing $10 \% \mathrm{FBS}$ at $37^{\circ} \mathrm{C}$ in $5 \% \mathrm{CO}_{2}$. The medium was exchanged every two days and the cells were digested with $0.1 \%$ pancreatin for subculture. The cellular morphology and apoptosis rate of unmodified BMSCs and BMSCs transfected with the survivin gene were detected for comparison. Subsequently, the BMSC populations were collected for injection into the animals.

Establishment of viral vector and transfection into BMScs. The viral vector (pLV-EX3d-P/hygro-EF1A-IRES-EmGFP, a GFP-marked vector) was purchased from Saiye (Guangzhou) Wusheng Technology Co., Ltd. (Guangzhou, China). The mouse survivin gene was cloned into the pLV-EX3d-P/hygro-EF1A-IRES-EmGFP viral vector. This successfully established the pLV-EX3d-P/hygro-EF1A-mSurvivin-IRES-EmGFP viral vector. This viral vector was transfected into BMSCs cultured in DMEM medium, followed by screening and amplification in the cells. The blank pLV-EX3d-P/hygro-EF1A-IRES-EmGFP vector was used to transfect the cells in the control group.

Pulmonary fibrosis model establishment. Fifty-four C57BL/6 mice with normal breeding were allocated at random into three groups: BMSC-survivin group (group A), BMSC group (group B) and the model group (group C). Anesthesia was induced by an intraperitoneal injection of $10 \%$ chloral hydrate (300 mg/kg). Then, the neck skin was disinfected and incised and $0.2 \mathrm{ml}$ bleomycin solution $(5 \mathrm{mg} / \mathrm{kg}$ ) was gradually administered dropwise into the trachea to induce the pulmonary fibrosis model. After $24 \mathrm{~h}$, mice in group A received $1 \times 10^{6}$ survivin gene-expressing BMSCs-in $200 \mu 1$ normal saline via the caudal vein, mice in group B received $1 \times 10^{6}$ BMSCs, while group $\mathrm{C}$ mice were treated with $200 \mu$ l normal saline. A selection of 6 mice from each group were sacrificed on days 7, 14 and 28, respectively. Following anesthesia, blood was collected from the vena jugularis externa, allowed to settle for $10 \mathrm{~min}$, centrifuged at $10,000 \mathrm{x}$ g for $10 \mathrm{~min}$ at $4^{\circ} \mathrm{C}$, then stored at $-80^{\circ} \mathrm{C}$. The left lung was used for pathological section, while the right lung was stored in liquid nitrogen for reverse transcription (RT)-qPCR and western blot analysis.

Analysis of pathology in lung tissue. Lung tissue was fixed in $4 \%$ paraformaldehyde for $24 \mathrm{~h}$, then subjected to dehydration, paraffin, embedding, sectioning and hematoxylin and eosin (H\&E) staining. Finally, the lung tissue was observed under a microscope in order to assess the degree of pulmonary fibrosis. The cells were also observed using a BX53 fluorescence microscope (Olympus Corporation, Tokyo, Japan).

SP-A determination in the lung tissue using immunohistochemistry. Lung tissue slices were dewaxed in xylene, treated with citric acid for antigen retrieval and then treated for $30 \mathrm{~min}$ with $\mathrm{H}_{2} \mathrm{O}_{2}$ to inactivate endogenous peroxidase. Slices were treated for $30 \mathrm{~min}$ with normal goat serum and incubated with a rabbit anti-mouse SP-A primary antibody (1:3,000; sc-7700; Santa Cruz Biotechnology, Inc.) overnight at $4^{\circ}$ C. Samples were rewarmed for $1 \mathrm{~h}$ at $37^{\circ} \mathrm{C}$, then incubated with a biotinylated goat anti-rabbit secondary antibody (1:2,000; sc-45101; Santa Cruz Biotechnology, Inc.) at $37^{\circ} \mathrm{C}$ for $30 \mathrm{~min}$. Next, streptavidin-biotin complex was added at $37^{\circ} \mathrm{C}$ for $30 \mathrm{~min}$, and the samples were stained using diaminobenzidine. Next, the samples were subjected to nuclear staining with hematoxylin, xylene dehydration, transparency (using Triton-X 100) and fixation with neutral balata. Images were obtained under a CX22 light microscope (Olympus Corporation) and analyzed using Image-Pro Plus software, version 2.0 (Media Cybernetics, Inc., Rockville, MD, USA). The average optical density (OD) represents the SP-A content.

Hydroxyproline content determination. Following anesthesia, blood was collected via the vena jugularis externa, allowed to stand for $10 \mathrm{~min}$, centrifuged for $10 \mathrm{~min}$ at a temperature of $4^{\circ} \mathrm{C}$, then kept at $-80^{\circ} \mathrm{C}$ until analysis. The hydroxyproline content was determined according to the instructions provided by the manufacturer of the hydroxyproline ELISA kit. OD values were obtained for each sample at $450 \mathrm{~nm}$. A standard curve was produced, and the concentration of hydroxyproline in each sample was determined from the standard curve, where the volume of the sample $(10 \mu \mathrm{l})$ was increased to $50 \mu \mathrm{l}$ with phosphate buffer solution.

Flow cytometry. Cell viability was evaluated by flow cytometry using Annexin V-fluorescein isothiocyanate (FITC) and propidium iodide (PI) double staining (Trevigen, Minneapolis, MN, USA). Briefly, the lung cells were suspended in Annexin V-FITC binding buffer and treated with Annexin V-FITC and PI at room temperature overnight. The stained cells were observed and analyzed using FACScan flow cytometry (Becton-Dickinson, Oxford UK).

$R T$ - $q P C R$ analysis. The total RNA of lung tissues was isolated using an RNAsimple Total RNA kit according to the manufacturer's instructions (Tiangen Biotech Co., Ltd., Beijing, China). RT was performed using the SuperScript ${ }^{\mathrm{TM}}$ III First-Strand Synthesis System (Invitrogen Life Technologies, Carlsbad, CA, USA) according to the manufacturer's instructions. The 


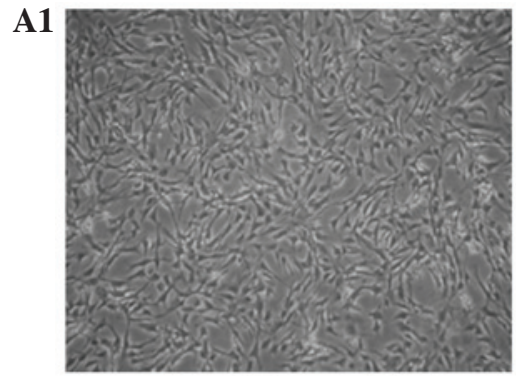

BMSC-survivin

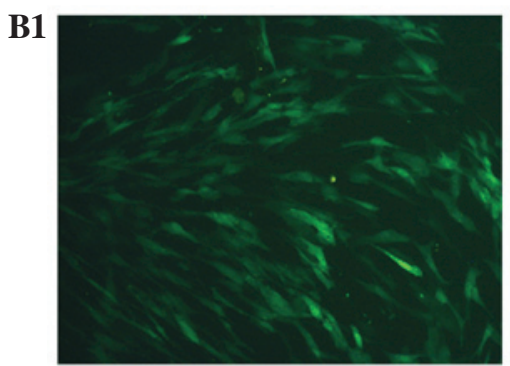

BMSC -survivin

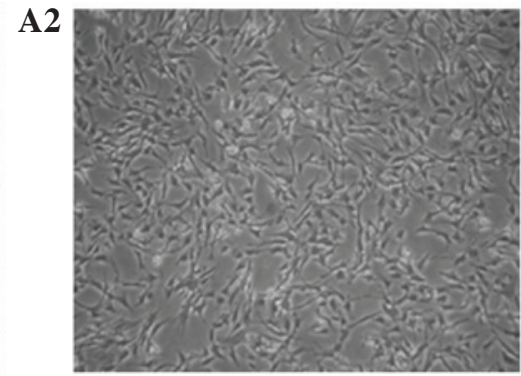

BMSC

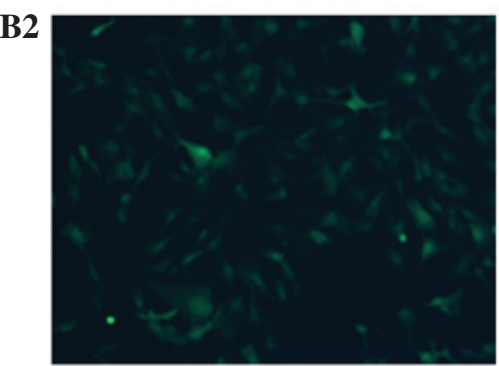

BMSC

Figure 1. Representative images of (A1) bone marrow-derived mesenchymal stem cells (BMSCs) transfected with survivin and (A2) normal BMSCs under a light microscope with no stain. Images of (B1) BMSCs with survivin and (B2) normal BMSCs captured using a fluorescence microscope. The images revealed green fluorescence due to the expression of green fluorescent protein. Magnification, x200.

procedure and conditions of the RT-qPCR analysis were as previously reported (4). RT-qPCR was performed using SYBR Premix Ex Taq II (Takara Biotechnology Co., Ltd., Dalian, China) using an ABI7500 Real-time PCR instrument (Applied Biosystems, Foster City, CA, USA). The products of the RT reaction were used for qPCR. Matrix metalloprotease (MMP)-9 and transforming growth factor (TGF)- $\beta 1$ levels were detected by qPCR. Primer-BLAST (http://www.ncbi. nlm.nih.gov/tools/primer-blast/) was used to design the primer sequences. The qPCR primer sequences were as follows: MMP-9, 5'-GCGCCACCACCGCCAACTAT-3' (upstream) and 5'-CTCGTGCGCTGCCACCAGAA-3' (downstream); TGF- $\beta 1$, 5'-TAACCGGCTGCTGACCCC-3' (upstream) and 5'-ATCCAGGGCTCTCCGGTGCC-3' (downstream); $\beta 1$-actin, 5'-AGGCTGTGCTGTCCCTGTATG-3' (upstream) and 5'-GAGGTCTTTACGGATGTCAACG-3' (downstream). Following qPCR, the products were subjected to $2 \%$ agarose gel electrophoresis and the resulting images were analyzed using Quantity One software (Bio-Rad Laboratories, Inc., Hercules, CA, USA), which calculated the gray area value of electrophoresis. The gray area ratios MMP-9/ $\beta$-actin and TGF $\beta-1 / \beta$-actin represent the relative quantities of MMP-9 and TGF- $\beta 1$, respectively.

Western blot assay. The lung tissues were isolated, snap-frozen in liquid nitrogen and immediately homogenized in ice-cold RIPA lysis buffer (Sigma-Aldrich, St. Louis, MO, USA) containing a cocktail of protease inhibitors (Roche, Basel, Switzerland). Caspase-3 and caspase-9 proteins were then detected using a western blot assay. Extracted total proteins (25 g) from lung tissue were resolved by SDS-PAGE, then transferred to a polyvinylidene difluoride membrane, which was blocked with 5\% skimmed milk powder solution for $1.5 \mathrm{~h}$. The membranes were treated with rabbit anti-mouse caspase- 3 monoclonal antibody (1:3,000; Santa Cruz Biotechnology, Inc.) and rabbit anti-mouse caspase- 9 antibody (1:4,000; Santa Cruz Biotechnology, Inc.) overnight at $4^{\circ} \mathrm{C}$. The membranes were washed, treated with goat anti-rabbit polyclonal secondary antibody (1:2,000; Santa Cruz Biotechnology, Inc.) for $2 \mathrm{~h}$ at room temperature, and then developed using an enhanced chemiluminescent (ECL) reagent (Thermo Scientific Pierce, Rockford, IL, USA). Analysis of the average OD value of images was performed using Quantity One software. The OD ratios of caspase-3/ $\beta$-actin and caspase- $9 / \beta$-actin represent the protein expression levels of caspase-3 and caspase-9, respectively.

Statistical analysis. Experimental data were analyzed using SPSS statistical software, version 17.0 (SPSS, Inc., Chicago, IL, USA). Measurement values are expressed as the mean \pm standard error of the mean. Inter-group comparisons were performed using one-way analysis of variance. $\mathrm{P}<0.05$ was considered to indicate a statistically significant difference.

\section{Results}

Morphology of BMSCs. BMSC distribution showed significant aggregation. Although the cells were predominantly single, 2-3-cell clusters of small cells were also present (Fig. 1). The BMSCs appeared ovular or cuboid in shape, some with a spindle shape on one side with a large nucleus which was circular or elliptic (Fig. 1). Cells with normal and uniform distribution presented with visible green fluorescence when observed under a fluorescence inverted microscope.

Survivin decreases the rate of apoptosis of BMSCs. The apoptosis rate of the BMSCs was detected using a flow cytometry assay with Annexin V-FITC and PI double staining. The results were that the apoptosis rate of BMSCs carrying the survivin 


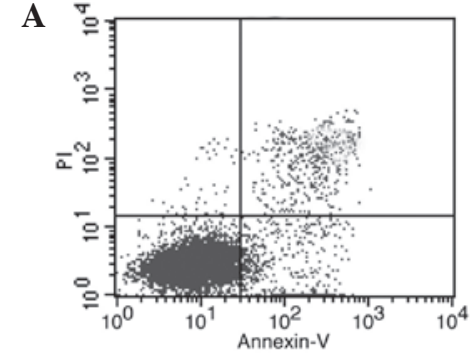

BMSC-survivin

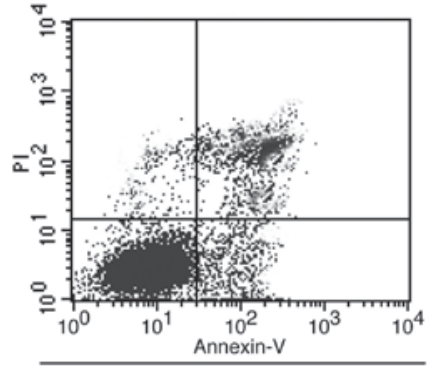

BMSC

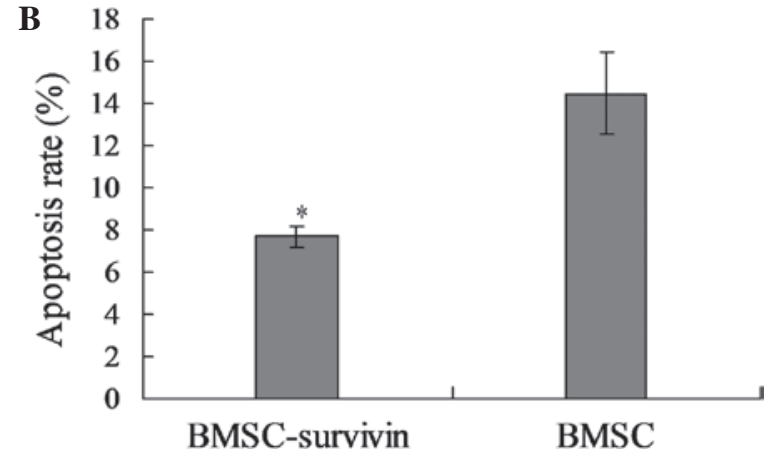

Figure 2. Apoptosis was detected in survivin-expressing and normal BMSCs. (A) Annexin V/PI double staining assays of cells transfected with survivin and untransfected cells. The y-axis indicates the number of PI-stained cells and the X-axis indicates the number of Annexin V-FITC-stained cells. (B) Quantitative analysis. The apoptosis rate of each preparation was evaluated from three independent experiments and represented as the mean \pm standard deviation. ${ }^{*} \mathrm{P}<0.05$ vs. the BMSC group. BMSC, bone marrow derived mesenchymal stem cell; PI, propidium iodide.
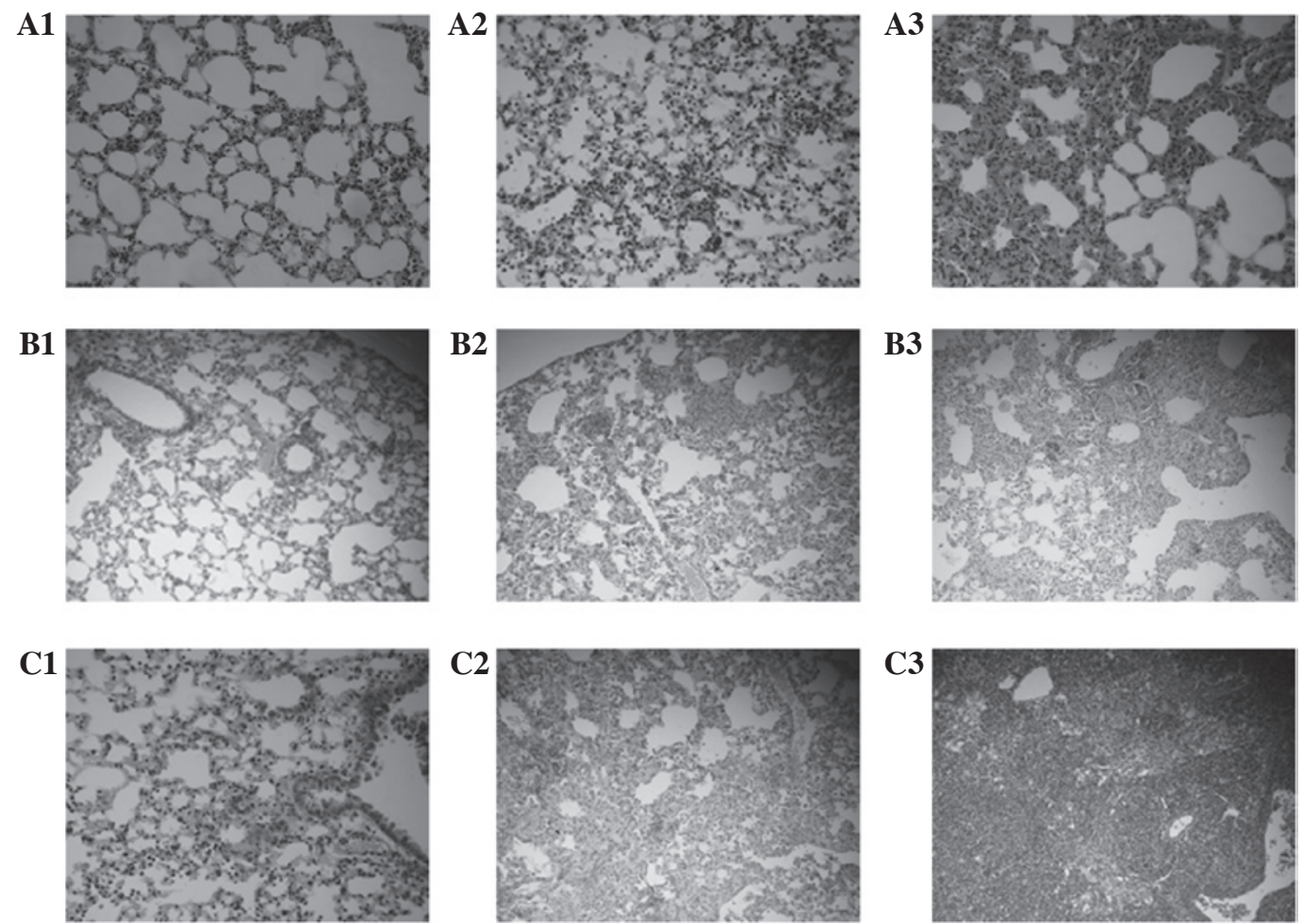

Figure 3. Hematoxylin and eosin staining of lung tissue in group A on (A1) day 7, (A2) day 14 and (A3) day 28; group B on (B1) day 7, (B2) day 14 and (B3) day 28; and group C on (C1) day 7, (C2) day 14 and (C3) day 28 (magnification, x200).

gene was $7.718 \pm 0.493 \%$, which was significantly reduced compared with that of the BMSC group (14.466 $\pm 1.953 \%$, $\mathrm{P}<0.05$; Fig. 2), indicating that the survivin gene reduces the apoptosis rate of BMSCs and prolongs the survival time of these cells in vitro.

Survivin enhances the anti-fibrotic effect of BMSCs. Fibrosis in group A was significantly inhibited compared with that in groups B and C at days 14 and 28 following treatment (Fig. 3). However, no significant differences were detected among the three groups at day 7 .

Survivin mitigates the inflammatory response in the alveoli. In group C, numerous inflammatory cells had infiltrated around the alveoli by day 7; on day 14, the alveolar interval had thickened, inflammatory cell infiltration was extensive, and the alveolar structure was observed to have collapsed; and on day 28 the alveolar structure was collapsed and degraded more seriously, and the alveolar spaces and intervals were occupied by collagen and fibrin. The alveolar inflammation in group B was less severe on day 7 compared with that in group $\mathrm{C}$. The amount of fibrin was also relatively less in group B than in group C. In group A, the inflammatory reaction was lighter than that in group B, and a small amount of fibrosis was distributed in the alveolar interval on the day 7. On day 28 , some of the alveolar structure had collapsed, there was a little collagen in the alveolar interval, and alveolar damage was rare (Fig. 4 and Table I).

Survivin decreases hydroxyproline levels in BMSCs. The ELISA results indicated statistically significant differences 

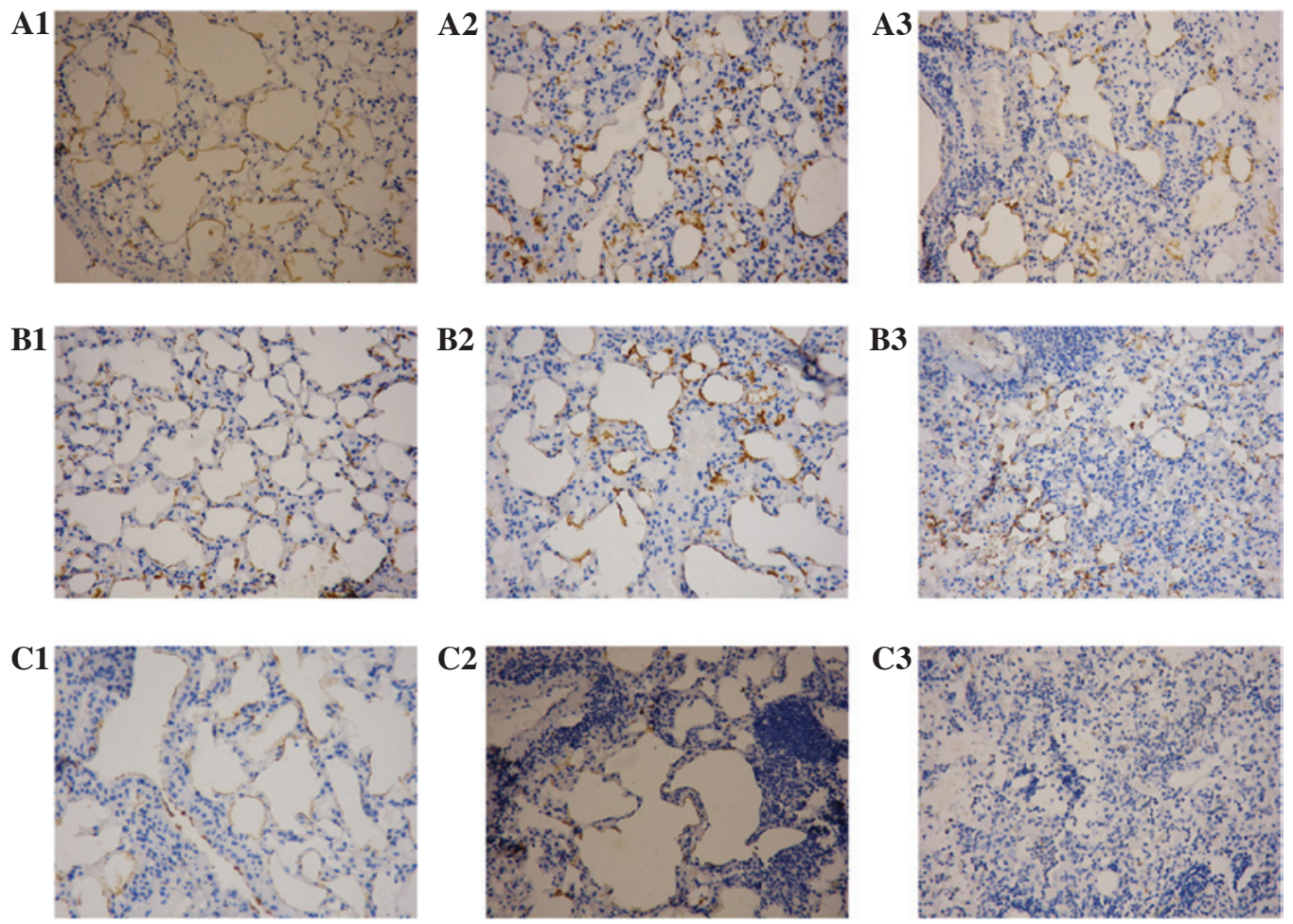

Figure 4. Immunohistochemical detection of surfactant protein A in the lung tissue in group A on (A1) day 7, (A2) day 14 and (A3) day 28; group B on (B1) day 7, (B2) day 14 and (B3) day 28; and group C on (C1) day 7, (C2) day 14 and (C3) day 28 (magnification, x200).

Table I. Comparison of mean optical density of surfactant protein A expression among the groups (mean \pm standard deviation).

\begin{tabular}{lccc}
\hline Group & Day 7 & Day 14 & \multicolumn{1}{c}{ Day 28 } \\
\hline A & $0.718 \pm 0.008^{\mathrm{a}, \mathrm{b}}$ & $0.643 \pm 0.013^{\mathrm{a}, \mathrm{b}}$ & $0.433 \pm 0.012^{\mathrm{a}, \mathrm{b}}$ \\
B & $0.669 \pm 0.013^{\mathrm{c}}$ & $0.550 \pm 0.024^{\mathrm{c}}$ & $0.372 \pm 0.009^{\mathrm{c}}$ \\
C & $0.581 \pm 0.007$ & $0.362 \pm 0.011$ & $0.277 \pm 0.013$ \\
\hline
\end{tabular}

${ }^{\mathrm{a}} \mathrm{P}<0.05$ vs. Group B; ${ }^{\mathrm{b}} \mathrm{P}<0.05$ vs. Group $\mathrm{C} ;{ }^{\mathrm{c}} \mathrm{P}<0.05$ vs. Group C.

Table II. Comparison of hydroxyproline content $(\mu \mathrm{g} / \mathrm{ml})$ among the groups (mean \pm standard deviation) as determined by ELISA.

\begin{tabular}{lccc}
\hline Group & Day 7 & Day 14 & Day 28 \\
\hline A & $0.106 \pm 0.002^{\mathrm{a}, \mathrm{b}}$ & $0.328 \pm 0.004^{\mathrm{a}, \mathrm{b}}$ & $0.457 \pm 0.006^{\mathrm{a}, \mathrm{b}}$ \\
B & $0.245 \pm 0.009^{\mathrm{c}}$ & $0.472 \pm 0.012^{\mathrm{c}}$ & $0.752 \pm 0.012^{\mathrm{c}}$ \\
C & $0.324 \pm 0.002$ & $0.794 \pm 0.007$ & $1.372 \pm 0.035$ \\
\hline
\end{tabular}

${ }^{\mathrm{a}} \mathrm{P}<0.05$ vs. Group B; ${ }^{\text {b }}<0.05$ vs. Group $\mathrm{C}$; ${ }^{\mathrm{C}} \mathrm{P}<0.05$ vs. Group C.

among the groups in the levels of hydroxyproline. At each time point the hydroxyproline levels in group A were reduced compared with those in group B, while group B hydroxyproline levels were reduced compared with those in group $\mathrm{C}$ (Table II).
Survivin suppresses the expression levels of TGF- $\beta 1$ and increases those of MMP-9. The expression levels of TGF- $\beta 1$ and MMP-9 mRNA were detected using an RT-qPCR assay. The expression levels of TGF- $\beta 1$ were significantly decreased in group A compared with groups $\mathrm{B}$ and $\mathrm{C}$ (Fig. 5; $\mathrm{P}<0.05$ ). Furthermore, the TGF- $\beta 1$ expression levels in group B were significantly decreased compared with those in group C (Fig. 5, $\mathrm{P}<0.05)$. The mRNA expression levels of MMP-9 in group A were significantly increased compared with those in groups B and $\mathrm{C}$ (Fig. 5; $\mathrm{P}<0.05$ ). Furthermore, the MMP-9 expression levels in group B were significantly increased compared with those in group $\mathrm{C}(\mathrm{P}<0.05)$.

Survivin increases the expression of caspase-3 and caspase-9. The expression levels of caspase- 3 and caspase- 9 were detected using a western blot assay. The results indicate that survivin significantly elevated caspase- 3 and caspase- 9 expression in group A compared with that in groups $\mathrm{B}$ and $\mathrm{C}$ (Fig. 6; $\mathrm{P}<0.05$ ). Furthermore, the levels of caspase- 3 and caspase- 9 in group B were significantly elevated compared with those in group $\mathrm{C}$ (Fig. 6; P<0.05).

\section{Discussion}

As the incidence of pulmonary fibrosis is increasing, a number of recent studies have indicated a range of factors that may cause damage to alveolar epithelial and endothelial cells. The abnormal repair response to this damage may lead to fibroblast activation and excessive accumulation of extracellular matrix (ECM) in the lung parenchyma $(7,8)$, which may ultimately lead to the occurrence of pulmonary fibrosis. At present, glucocorticoids are the preferred drug treatment for pulmo- 
A

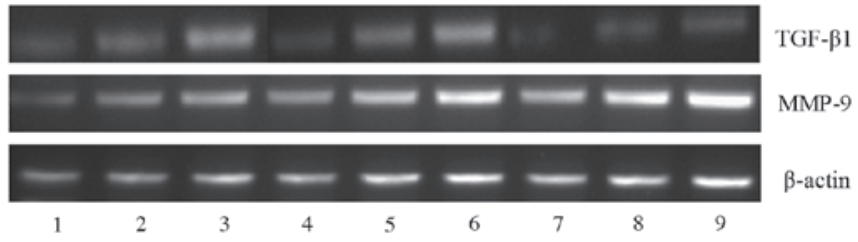

B
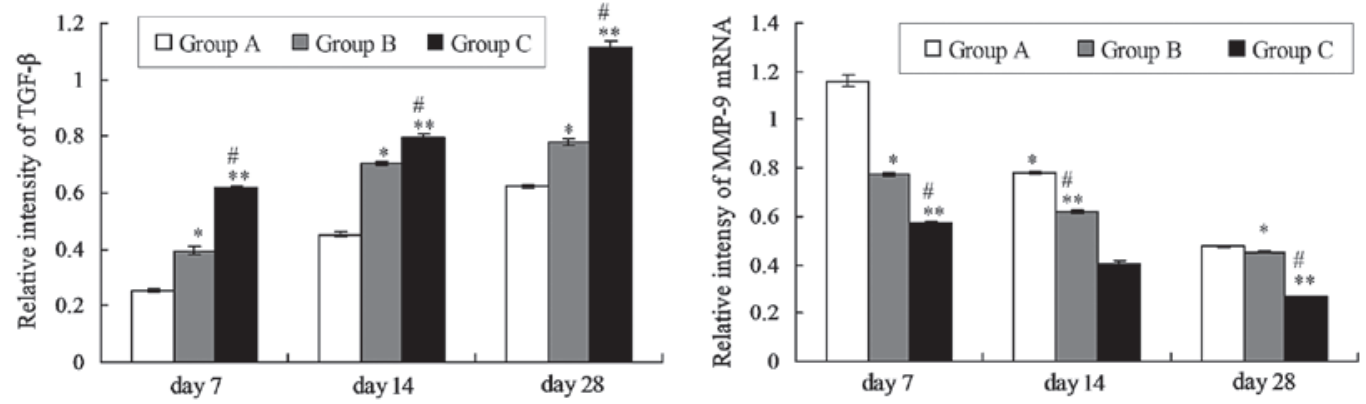

Figure 5. Reverse transcription quantitative polymerase chain reaction (qPCR) analysis of mRNA expression levels of MMP-9 and TGF- $\beta 1$. qPCR analysis of (A) MMP-9 and TGF- 31 mRNA expression in group A on (lane 1) day 7, (lane 2) day 14 and (lane 3) day 28; group B on (lane 4) day 7, (lane 5) day 14 and (lane 6) day 28; and group C on (lane 7) day 7, (lane 8) day 14 and (lane 9) day 28. (B) Quantitative analysis of the relative intensities of TGF- $\beta 1$ and MMP-9 among the groups. ${ }^{*} \mathrm{P}<0.05,{ }^{* *} \mathrm{P}<0.01$ vs. group $\mathrm{A} .{ }^{.} \mathrm{P}<0.05$ vs. group $\mathrm{B}$. TGF- $\beta 1$, transforming growth factor- $\beta 1$; MMP-9, matrix metalloproteinase-9.

A

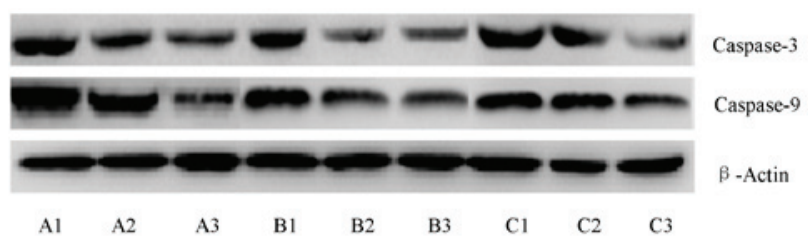

B
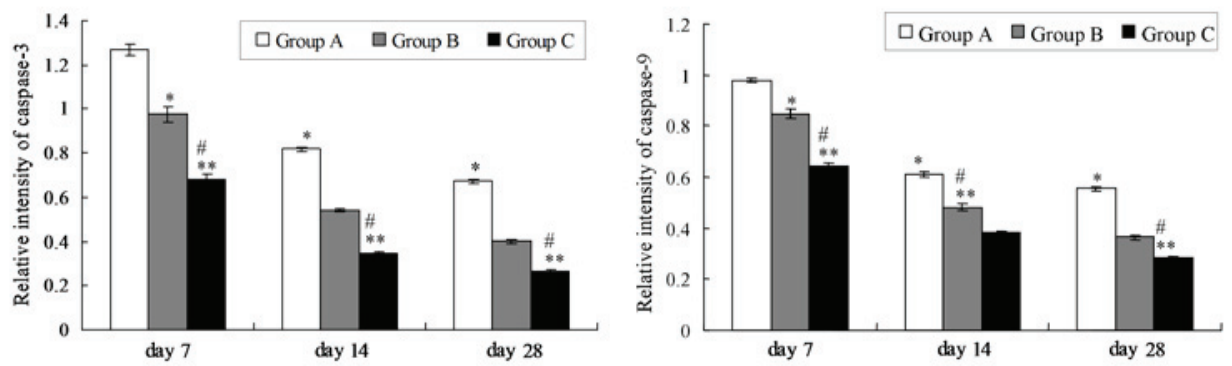

Figure 6. Caspase-3 and caspase-9 protein content of lung determined by western blot analysis. Western blotting results for (A) caspase-3 and caspase-12 protein expression in group A on (lane 1) day 7, (lane 2) day 14 and (lane 3) day 28; group B on (lane 4) day 7, (lane 5) day 14 and (lane 6) day 28; and group C on (lane 7) day 7, (lane 8) day 14 and (lane 9) day 28. (B) Quantitative analysis of the relative intensities for caspase-3 and caspase-12. ${ }^{*}<0.05$, ${ }^{* *} \mathrm{P}<0.01$ vs.

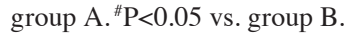

nary fibrosis; however, the curative effect of glucocorticoids is not sufficient to reverse or retard the course of pulmonary fibrosis. Furthermore, the long-term use of glucocorticoids may result in a series of complications, such as bacterial or fungal lung infection and metabolic disorders. A number of previous studies have investigated the use of agents targeting cytokines (9), such as pirfenidone, imatinib and interferon (IFN) (10), particularly IFN- $\alpha$, as a treatment for pulmonary fibrosis. Liu et al (11) reported that IFN- $\alpha$ may inhibit the expression of TGF- $\beta 1$ and connective tissue growth factor, and thus reduce the generation of ECM in a bleomycin-induced model of pulmonary fibrosis. However, IFN- $\alpha$ is an expensive treatment, which limits its potential for widespread use. In addition, IFN- $\alpha$ exhibits a number of potential side-reactions.
$\mathrm{N}$-acetyl cysteine is able to notably increase glutathione levels in the lung tissue and exhibits strong resistance to oxidation and cell detoxification. Hence, $\mathrm{N}$-acetyl cysteine may improve the lung function of patients with idiopathic pulmonary fibrosis and is well tolerated, but is not able to reduce mortality (12). Lung transplantation is the primary method for treating end-stage pulmonary fibrosis and can markedly improve the lung function of patients (13); however, lung transplantation is not applicable to a wide range of clinics. Certain traditional Chinese medicines may exert curative effects against lung fibrosis (14), but the underlying mechanisms of these treatments require further study. Thus, the treatment of pulmonary fibrosis remains challenging, and a safer and more effective therapy for pulmonary fibrosis is urgently required. 
A number of studies have demonstrated that BMSCs are able to grow and participate in the development of lung tissue. The administration of BMSCs has been shown to ameliorate fibrotic injuries, suggesting the possibility that stem cell-based therapies may be developed as an effective intervention against pulmonary fibrosis (15-18). BMSCs exist in numerous types of human tissue, possess multidirectional differentiation potential and may be obtained from a variety of tissues. Currently, the studies investigating BMSC separation, differentiation, expansion, immune phenotype, features and mechanism, including immune regulation and anti-inflammatory function, have increased significantly (19). Previous studies have confirmed that exogenous BMSCs may be transplanted into impaired lung tissue, and are able to differentiate into alveolar type II epithelial cells, which possess a higher migration rate in the early stages of lung injury $(1,18,20)$. Furthermore, a previous animal study confirmed that BMSCs are able to develop in lung tissue afflicted with bleomycin-induced pulmonary fibrosis, and to reduce the extent of the pulmonary fibrosis (21).

The primary function of the survivin gene is to inhibit cell division and apoptosis. Survivin is the regulatory gene of the cell cycle in the G2/M phase, causing cells to exit the G2/M phase checkpoint and accelerate conversion to the $\mathrm{S}$ phase, in addition to inhibiting stationary $\mathrm{G}$ phase. Furthermore, survivin participates in the regulation of cellular mitosis by binding with the microtubule proteins of the mitotic spindle (22), promoting the abnormal proliferation of transformed cells and mitigating the occurrence of apoptosis. Furthermore, previous studies have reported that survivin is associated with tumor angiogenesis and multiple drug resistance $(23,24)$. Therefore, it may be speculated that survivin enhances the antifibrotic effect of BMSCs by inhibiting the occurrence of apoptosis.

In the present study, BMSCs transfected with survivin were transferred into the lung tissue of mice modeling pulmonary fibrosis as a result of treatment with bleomycin $24 \mathrm{~h}$ previously. This was conducted to evaluated the hypothesis that the apoptosis of such BMSCs may be inhibited and the repair of damaged lung tissue promoted, thus mitigating the progression of pulmonary fibrosis.

The results of the present experiments indicate that the BMSCs containing GFP and survivin were successfully transplanted into the mice modeling bleomycin-induced pulmonary fibrosis. Green fluorescence was detected in the lung tissue, demonstrating that the BMSCs targeted damaged lung tissue. The degree of pulmonary fibrosis in group B was evidently reduced compared with that in group $\mathrm{C}$, while the damage in group A was lessened compared with that in group B. Immunohistochemical detection showed that SP-A expression in group B was increased compared with that in group C, while SP-A expression in group A was higher compared with that in group B. Collectively, these results indicate that BMSCs may differentiate into epithelial cells and alveolar type II epithelial cells in damaged lung tissue, participate in the repair of lung injury and reduce pulmonary fibrosis, which is consistent with the previous results of Chen et al (25). In vitro, the results of flow cytometry indicate that the apoptosis rate of BMSCs transfected with survivin was significantly lower compared with that of BMSCs that were not transfected with survivin. Fluorescence intensity in the lung tissue of group A was markedly increased compared with that in group B. Western blot analysis results indicate that the protein expression levels of caspase-3 and caspase-9, which are effectors in the functional pathway of the survivin gene, were increased in group A compared with the levels in the other two groups, indicating that the expression of survivin gene was increased in group A. Hence, the survivin gene may delay BMSC apoptosis in vivo and in vitro, and sequentially block the process of pulmonary fibrosis in the initial stages.

The RT-qPCR results suggest that the mRNA expression levels of MMP-9 were increased and those of TGF- $\beta 1$ were reduced in group A compared with those in group B. Since MMP-9 and TGF- $\beta 1$ are crucial factors associated with pulmonary fibrosis $(3,26)$, these results demonstrate that BMSCs, particularly when transfected with survivin, may be able to reduce bleomycin-induced pulmonary fibrosis in mice, which is consistent a previous report (21). Furthermore, previous studies have indicated that BMSCs secrete a variety of cell factors that participate in vascular regeneration and endothelial cell remodeling in lung tissue, and these factors may induce BMSCs to differentiate $(6,27)$. In addition, it has been reported that BMSCs are able to promote the expression of growth factors in local damaged tissue, inhibiting cell apoptosis, reinforcing cell repair and promoting cell proliferation and differentiation (28). However, further studies are required to confirm the present and previous findings.

BMSCs possess multidirectional differentiation potential; a previous study suggested that stem cells contain gene sequences that resemble those of cancer cells (29). Human mesenchymal stem cells may transform spontaneously and exhibit long-term proliferation and differentiation in vivo (30). These observations suggest that ensuring the safety assessment of BMSC-based treatment is crucial (31). Survivin is expressed in the majority of tumor tissues, in which it is able to inhibit tumor cell apoptosis and induce cancer cells to proliferate and differentiate constantly, thus enhancing tumor growth. In addition, survivin may cause loss of the normal cell cycle checkpoint, leading to disorders of the normal cell cycle (32).

In summary, the results of the present study indicate that BMSCs are effective in preventing bleomycin-induced lung fibrosis, and that survivin is able to enhance the protective effects of BMSCs. However, further safety assessments are required to evaluate the potential clinical value of this intervention, including further investigation into cancerous characteristics of the survivin gene.

\section{References}

1. Liu X, Qian L, Nan HY, Cui M, Hao XY and Du YF: Function of the transforming growth factor- $\beta$ 1/c-Jun N-terminal kinase signaling pathway in the action of thalidomide on a rat model of pulmonary fibrosis. Exp Ther Med 7: 669-674, 2014.

2. Moodley Y, Atienza D, Manuelpillai U, Samuel CS, Tchongue J, Ilancheran S, Boyd R and Trounson A: Human umbilical cord mesenchymal stem cells reduce fibrosis of bleomycin-induced lung injury. Am J Pathol 175: 303-313, 2009.

3. Selman M, King TE and Pardo A: Idiopathic pulmonary fibrosis: prevailing and evolving hypotheses about its pathogenesis and implications for therapy. Ann Intern Med 134: 136-151, 2001

4. Ortiz LA, Gambelli F, McBride C, Gaupp D, Baddo M, Kaminski $\mathrm{N}$ and Phinney DG: Mesenchymal stem cell engraftment in lung is enhanced in response to bleomycin exposure and ameliorates its fibrotic effects. Proc Natl Acad Sci USA 100: 8407-8411, 2003. 
5. Zhang ZH, Lu Y, Luan Y and Zhao JJ: Effect of bone marrow mesenchymal stem cells on experimental pulmonary arterial hypertension. Exp Ther Med 4: 839-843, 2012.

6. Kondo K, Shintani S, Shibata R, Murakami H, Murakami R, Imaizumi M, Kitagawa Y and Murohara T: Implantation of adipose-derived regenerative cells enhances ischemia-induced angiogenesis. Arterioscler Thromb Vasc Biol 29: 61-66, 2009.

7. Tzouvelekis A, Harokopos V, Paparountas T, Oikonomou N, Chatziioannou A, Vilaras G, Tsiambas E, Karameris A, Bouros D and Aidinis V: Comparative expression profiling in pulmonary fibrosis suggests a role of hypoxia-inducible factor- $1 \alpha$ in disease pathogenesis. Am J Respir Crit Care Med 176: 1108-1119, 2007.

8. Wu Z, Wang X, Yang R, Liu Y, Zhao W, Si J, Ma X, Su C, Liu Y, Tan Y, Liu W, et al: Effects of carbon ion beam irradiation on lung injury and pulmonary fibrosis in mice. Exp Ther Med 5: 771-776, 2013

9. Sun YW, Li BP, Wang Xuan, Zhang L, Lu PY and Zhao XY: Oriented migration of intravenously administrated mesenchymal stem cells transfected with adenovirus vector mediated green fluorescence protein in the lung tissue of pulmonary emphysema rats. Zhongguo Zu Zhi Gong Cheng Yan Jiu Yu Lin Chuang Kang Fu 14: 2528-2532, 2010 (In Chinese).

10. Cai Y: Pulmonary fibrosis drug treatment progress. Shi Jie Lin Chuang Yao Wu 29: 17-19, 2008 (In Chinese).

11. Liu T, Xie M, Wang HL and Ling W: Effect of matrine and IFN- $\gamma$ on bleomycin-induced pulmonary fibrosis. Nanjing Yi Ke Da Xue Xue Bao 28: 592-596, 2008 (In Chinese).

12. Bhatt N, Baran C, Allen J, Magro C and Marsh CB: Promising pharmacologic innovations in treating pulmonary fibrosis. Curr Opin Pharmacol 6: 284-292, 2006.

13. Lettieri CJ, Veerappan GR, Helman DL, Mulligan CR and Shorr AF: Outcomes and safety of surgical lung biopsy for interstitial lung disease. Chest 127: 1600-1605, 2005.

14. $\mathrm{Hu} \mathrm{F}$ : Research progress of Chinese medicine treatment of pulmonary fibrosis. Yi Yao Dao Bao 11: 1471-1474, 2011 (In Chinese).

15. Xu L, Xiao W, Ma DD, Zhou SY and Zhang QH: Ulcerative colitis combined with acute interstitial lung disease and airway disease: A case report and literature review. Exp Ther Med 8: 1229-1236, 2014.

16. Kotton DN, Ma BY, Cardoso WV, Sanderson EA, Summer RS, Williams MC and Fine A: Bone marrow-derived cells as progenitors of lung alveolar epithelium. Development 128: 5181-5188, 2001

17. Krause DS, Theise ND, Collector MI, Henegariu O, Hwang S, Gardner R, Neutzel S and Sharkis SJ: Multi-organ, multi-lineage engraftment by a single bone marrow-derived stem cell. Cell 105 369-377, 2001.

18. Teke T, Maden E, Kiyici A, Korkmaz C, Gok Z, Ozer F, Imecik O and Uzun K: Cigarette smoke and bleomycin-induced pulmonary oxidative stress in rats. Exp Ther Med 4: 121-124, 2012.
19. Tzouvelekis A, Antoniadis A and Bouros D: Stem cell therapy in pulmonary fibrosis. Curr Opin Pulm Med 17: 368-373, 2011.

20. Cui A, Dai HP and Dai JW: Effects of bone marrow mesenchymal stem cells on bleomycin induced pulmonary fibrosis in rats. Zhonghua Jie He He Hu Xi Za Zhi 30: 677-682, 2007 (In Chinese)

21. Rojas M, Xu J, Woods CR, Spears W, Roman J and Brigham KL: Bone marrow-derived mesenchymal stem cells in repair of the injured lung. Am J Respir Cell Mol Biol 33: 145-152, 2005.

22. Beardmore VA, Ahonen LJ, Gorbsky GJ and Kallio MJ: Survivin dynamics increases at centromeres during $\mathrm{G} 2 / \mathrm{M}$ phase transition and is regulated by microtubule-attachment and Aurora B kinase activity. J Cell Sci 117: 4033-4042, 2004.

23. Yang M, Liu Y, Lu S, Wang Z, Wang R, Zi Y and Li J: Analysis of the expression levels of surviving and VEGF in patients with acute lymphoblastic leukemia. Exp Ther Med 5: 305-307, 2013.

24. Liu F, Liu S, He S, Xie Z, Zu X and Jiang Y: Survivin transcription is associated with P-glycoprotein/MDR1 over expression in the multidrug resistance of MCF-7 breast cancer cells. Oncol Rep 23: 1469-1475, 2010

25. Chen Y, Ma N, Mei J, Xiao HB, Lu SW, Xu HY and Zhong H: In vitro induction of human bone marrow mesenchymal stem cells to differentiate into type II alveolar epithelial cells. Zhong Guo Zu Zhi Gong Cheng Yan Jiu 16: 1737-1741, 2012 (In Chinese).

26. Guo T, Fang M, Zhang D and Li X: Combination treatment with asiaticoside and repamycin: A new hope for in-stent restenosis. Exp Ther Med 6: 557-561, 2013.

27. Jiang PC, Xiong WP, Wang G, Ma C, Yao WQ, Kendell SF, Mehling BM, Yuan XH and Wu DC: A clinical trial report of autologous bone marrow-derived mesenchymal stem cell transplantation in patients with spinal cord injury. Exp Ther Med 6: 140-146, 2013.

28. Rubio D, Garcia S, Paz MF, De la Cueva T, Lopez-Fernandez LA, Lloyd AC, Garcia-Castro J and Bernad A: Molecular characterization of spontaneous mesenchymal stem cell transformation. PLoS One 3: e1398, 2008.

29. de la Fuente R, Bernad A, Garcia-Castro J, Martin MC and Cigudosa JC: Retraction: Spontaneous human adult stem cell transformation. Cancer Res 70: 6682, 2010.

30. Lin ZX and $\mathrm{Xu} \mathrm{H}$ : Current understanding into the biological characteristics of mesenchymal stem cells. Zhongguo $\mathrm{Zu} \mathrm{Zhi}$ Gong Cheng Yan Jiu Yu Lin Chuang Kang Fu 15: 3577-3580, 2011 (In Chinese).

31. Wang LZ, Xu L, Tan DM and Tan Y: The progress of survivin. Yi Xue Zong Shu 12: 1089-1092, 2006 (In Chinese).

32. Kobayashi M, Huang CL, Sonobe M, Kikuchi R, Ishikawa M, Kitamura J, Miyahara R, Menju T, Iwakiri S, Itoi K, Yasumizu R and Date H: Intratumoral Wnt2B expression affects tumor proliferation and survival in malignant pleural mesothelioma patients. Exp Ther Med 3: 952-958, 2012. 